ja0624319 - Supplemental information

\title{
When water is not water? Exploring water confined in reverse micelles using a highly charged inorganic molecular probe
}

Bharat Baruah, Jennifer M. Roden, Myles Sedgwick, N. Mariano Correa, $\dagger$ Debbie C.

$$
\text { Crans, * and Nancy E. Levinger* }
$$

Department of Chemistry, Colorado State University, Colorado 80523-1872, USA

* corresponding authors: email Debbie.Crans@,ColoState.edu, Nancy.Levinger@ColoState.edu

† permanent address Departamento de Química. Universidad Nacional de Río Cuarto. Agencia Postal \# 3. C.P. 5800 Río Cuarto. Argentina.

\section{SI. Experimental Methods}

SI. A. Materials. Sodium metavanadate, $\mathrm{NaVO}_{3}$ and AOT (sodium bis(2ethylhexyl)sulfosuccinate) were purchased from Aldrich. AOT was purified by dissolving in methanol and stirring it overnight in the presence of activated charcoal. Subsequent filtration and removal of methanol by distillation under vacuum yielded AOT suitable for use. Isooctane was received from Aldrich and was used without further purification. Deionized water was used throughout and prepared to a specific resistivity of $>18 \mathrm{M} \Omega$ cm (Barnstead E-pure system). 
SI. B. Decavanadate Solution Preparation. A series of $10 \mathrm{mM}$ decavanadate $\left(\mathrm{V}_{10}\right)$ stock solutions $\left(100 \mathrm{mM} \mathrm{NaVO}_{3}\right)$ were prepared from $\mathrm{pH} 3$ to $8 . \mathrm{V}_{10}$ solutions were prepared by dissolving $\mathrm{NaVO}_{3}$ into water in a volumetric flask, adjusting the $\mathrm{pH}$ to 3 using $6 \mathrm{M} \mathrm{HCl}$ to form $\mathrm{V}_{10}$ before raising the $\mathrm{pH}$ to the required value using $1 \mathrm{M} \mathrm{NaOH}$ and the required volume. All solutions were freshly prepared. The $\mathrm{pH}$ values of the aqueous solutions were measured at $25^{\circ} \mathrm{C}$ using a $\mathrm{pH}$ meter (Orion 420A) calibrated with three buffers of $\mathrm{pH} 4,7$ and 10 . The $\mathrm{pH}$ values were adjusted to within $0.05 ; \mathrm{pH}$ variability between sample preparation and measurement was within $0.1 \mathrm{pH}$ unit unless otherwise noted.

SI. C. RMs Preparation and Characterization. A $0.5 \mathrm{M}$ AOT stock solution was prepared by dissolving AOT in isooctane under ambient conditions. Decavanadate-loaded RMs of a specific $w_{0}$ value, routinely 12,16 , and 20 , were prepared by pipetting a specific volume of the aqueous $\mathrm{NaVO}_{3}$ stock solutions at a known $\mathrm{pH}$ into aliquots of the AOT stock solution. All samples were mixed by vortexing 2-3 min prior to ${ }^{51} \mathrm{~V}$ NMR spectroscopic measurements. The resulting solutions were clear and yellow. Dynamic light scattering experiments were performed to identify the presence RMs in solution and to measure their size and polydispersity (Wyatt, DynaPro Titan). All the measurements were performed at $25^{\circ} \mathrm{C}$. Viscosities used in the particle size calculations were obtained from experimental measurements the samples using Ubbeholde viscometers. For AOT concentrations at or below $0.2 \mathrm{M}$, viscosities were the same as pure isooctane within the error of the measurement. Sizes measured were similar to those reported in the literature. ${ }^{52-54}$ Samples were also characterized by their conductivity using a conductivity meter (Orion 150A) equipped with a glass cell with two rectangular Pt electrodes (15 mm 
x $10 \mathrm{~mm}$ ) with cell constant 0.1 . The conductivity cell was calibrated with a standard 100 $\mu \mathrm{S} / \mathrm{cm}$ solution.

SI. D. ${ }^{51} V$ NMR Spectroscopy. ${ }^{51} \mathrm{~V}$ NMR spectra were recorded using a Varian INOVA-300 spectrometer at $78.9 \mathrm{MHz} .{ }^{51} \mathrm{~V}$ NMR spectra were acquired with a $83.6 \mathrm{kHz}$ spectral window, a $60^{\circ}$ pulse angle, and a $0.096 \mathrm{~s}$ acquisition time with no relaxation delay. ${ }^{51} \mathrm{~V}$ chemical shifts were referenced against an external sample of $\mathrm{VO}_{4}{ }^{3-}$, which had been referenced against a sample of $\mathrm{VOCl}_{3}$. For peak position measurements, a 15 $\mathrm{Hz}$ exponential line broadening was applied before Fourier transformation. The relative intensities of the vanadium oligomer signals were used to calculate the concentrations of decavanadate $\left(\mathrm{V}_{10}\right)$ and other oligomeric vanadates $\left(\mathrm{V}_{1}\right.$ monomer; $\mathrm{V}_{2}$ dimer; $\mathrm{V}_{4}$ tetramer and $V_{5}$ pentamer). ${ }^{44}$

SI. E. Spectral Analysis and Curve Fitting of Spectra. The NMR FIDs collected were Fourier transformed and subjected to phase correction (MestReC V. 4.5.9.1 NMR data processing software for Windows). Resulting spectral peaks were fitted to find chemical shifts and linewidths (OriginPro 7). To gauge the intensity of each spectral peak, when needed spectra were background subtracted before fitting using a Lorentzian line shape function. Fits to spectral peaks yielded peak positions, linewidths and integrated intensities. 


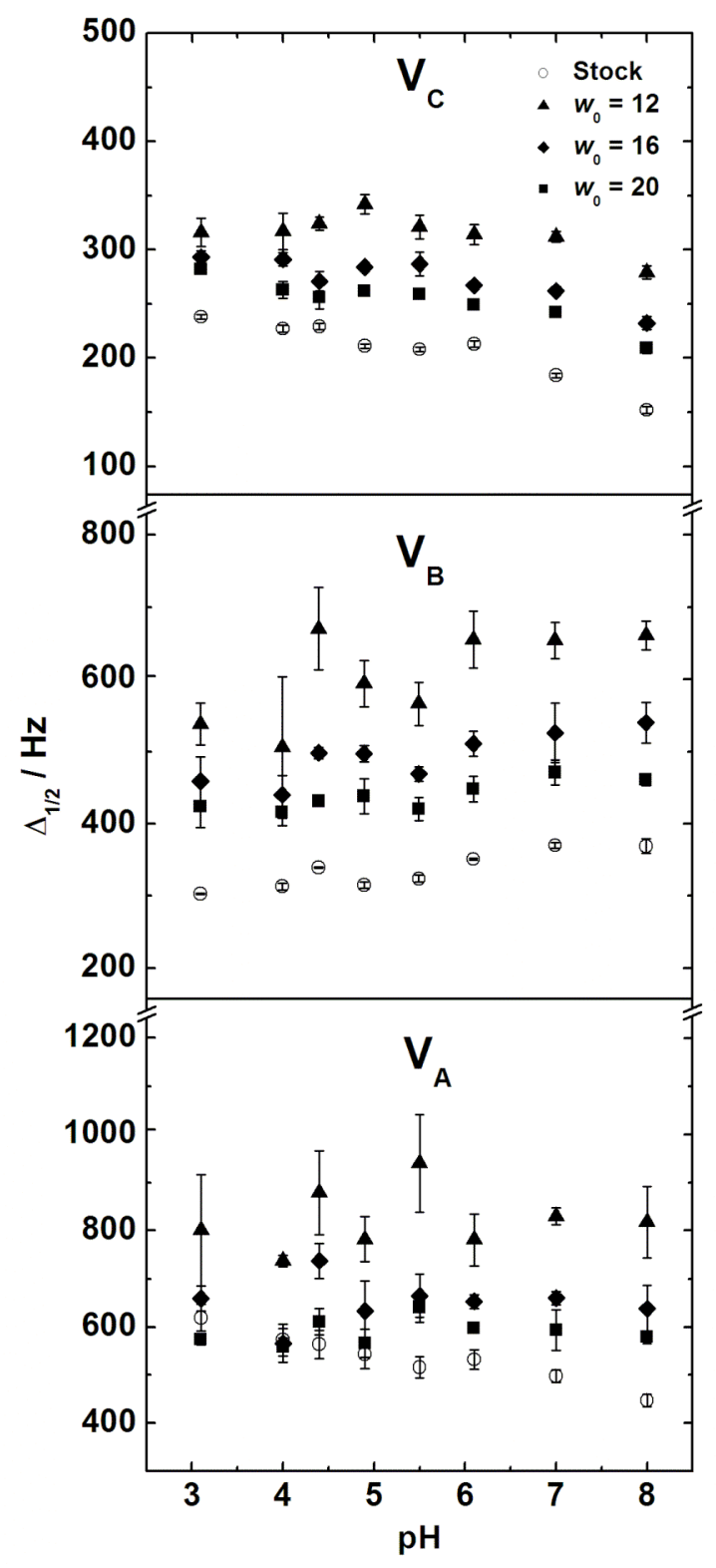

Figure S1: ${ }^{51} \mathrm{~V}$ NMR linewidths as a function of $\mathrm{pH}$ for each vanadium atom type $\left(\mathrm{V}_{\mathrm{C}}\right.$, $\mathrm{V}_{\mathrm{B}}$ and $\mathrm{V}_{\mathrm{A}}$ ) in $\mathrm{V}_{10}$. 
ja0624319 - Supplemental information

When water is not water? Exploring water confined in reverse micelles using a highly charged inorganic molecular probe Bharat Baruah, Jennifer M. Roden, Myles Sedgwick, N. Mariano Correa, $\uparrow$ Debbie C. Crans, ${ }^{*}$ and Nancy E. Levinger* Department of Chemistry, Colorado State University, Colorado 80523-1872, USA

* corresponding authors: email Debbie.Crans@ColoState.edu, Nancy.Levinger@ColoState.edu

† permanent address Departamento de Química. Universidad Nacional de Río Cuarto. Agencia Postal \# 3. C.P. 5800 Río Cuarto. Argentina. 
Table S1. ${ }^{51} \mathrm{~V}$ NMR chemical shift data for $\mathrm{V}_{\mathrm{A}}, \mathrm{V}_{\mathrm{B}}$ and $\mathrm{V}_{\mathrm{C}}$ in a $100 \mathrm{mM} \mathrm{NaVO}_{3}$ aqueous stock solution (of $\mathrm{pH}$ 3-8) and their corresponding reverse micellar (0.5 M AOT / isooctane) solution of $w_{0}=12,16$ and 20.

\begin{tabular}{|c|c|c|c|c|c|c|c|c|c|c|c|c|}
\hline \multirow[b]{2}{*}{$\mathrm{pH}$} & \multicolumn{4}{|c|}{$\mathbf{V}_{\mathrm{A}}$} & \multicolumn{4}{|c|}{$\mathbf{V}_{\mathbf{B}}$} & \multicolumn{4}{|c|}{$\mathbf{V}_{\mathbf{C}}$} \\
\hline & Stock & $w_{0}=12$ & $w_{0}=16$ & $w_{0}=20$ & Stock & $w_{0}=12$ & $w_{0}=16$ & $w_{0}=20$ & Stock & $w_{0}=12$ & $w_{0}=16$ & $w_{0}=20$ \\
\hline 3.1 & $-425.3 \pm 1.0$ & $-423.4 \pm 1.0$ & $-424.1 \pm 0.3$ & $-424.1 \pm 0.2$ & $-507.4 \pm 0.1$ & $-501.0 \pm 0.1$ & $-501.6 \pm 0.1$ & $-502.0 \pm 0.3$ & $-525.3 \pm 0.2$ & $-516.3 \pm 0.1$ & $-517.0 \pm 0.1$ & $-517.7 \pm 0.3$ \\
\hline 4.0 & $-424.7 \pm 0.4$ & $-423.7 \pm 0.4$ & $-424.0 \pm 0.3$ & $-423.9 \pm 0.1$ & $-504.7 \pm 1.0$ & $-501.0 \pm 0.3$ & $-501.1 \pm 0.2$ & $-501.2 \pm 0.1$ & $-521.5 \pm 1.0$ & $-516.3 \pm 0.2$ & $-516.4 \pm 0.2$ & $-516.6 \pm 0.1$ \\
\hline 4.4 & $-424.5 \div 0.02$ & $-424.2 \mp 0.1$ & $-423.8 \div 1.0$ & $-424.1 \div 0.1$ & $-503.2 \div 0.3$ & $-500.8 \div 0.1$ & $-501.1 \mp 0.1$ & $-501.2 \pm 0.1$ & $-519.7 \mp 0.2$ & $-516.2 \div 0.03$ & $-516.1 \mp 0.01$ & $-516.4 \div 0.04$ \\
\hline 4.9 & $-424.0 \pm 1.0$ & $-423.9 \pm 0.2$ & $-423.5 \pm 0.2$ & $-423.6 \pm 0.2$ & $-501.8 \pm 0.1$ & $-500.7 \pm 0.2$ & $-500.6 \pm 0.1$ & $-500.7 \pm 0.2$ & $-517.4 \pm 0.1$ & $-516.0 \pm 0.1$ & $-515.9 \pm 0.1$ & $-516.0 \pm 0.1$ \\
\hline 5.5 & $-423.8 \pm 1.0$ & $-423.1 \pm 0.2$ & $-423.9 \pm 1.0$ & $-423.9 \pm 0.2$ & $-500.9 \pm 0.1$ & $-500.5 \pm 0.2$ & $-500.3 \pm 0.1$ & $-500.7 \pm 0.1$ & $-516.2 \pm 0.2$ & $-515.9 \pm 0.2$ & $-516.0 \pm 0.1$ & $-516.0 \pm 0.1$ \\
\hline 6.1 & $-423.9 \pm 0.4$ & $-423.7 \pm 0.4$ & $-423.8 \pm 1.0$ & $-423.9 \pm 0.3$ & $-500.4 \pm 0.3$ & $-500.4 \pm 0.04$ & $-500.6 \pm 0.2$ & $-500.5 \pm 0.1$ & $-515.6 \pm 0.2$ & $-515.8 \pm 0.04$ & $-515.7 \pm 0.1$ & $-515.7 \pm 0.1$ \\
\hline 7.0 & $-423.3 \pm 0.01$ & $-423.9 \pm 0.6$ & $-423.6 \pm 0.4$ & $-423.5 \pm 0.2$ & $-498.6 \pm 0.1$ & $-499.3 \pm 0.04$ & $-499.2 \pm 0.1$ & $-499.2 \pm 0.1$ & $-514.4 \pm 0.03$ & $-514.8 \pm 0.03$ & $-514.7 \pm 0.1$ & $-514.7 \pm 0.1$ \\
\hline 8.0 & $-422.7 \pm 0.1$ & $-423.0 \pm 0.2$ & $-423.0 \pm 0.2$ & $-423.1 \pm 0.1$ & $-497.0 \pm 0.1$ & $-498.3 \pm 0.1$ & $-498.1 \pm 0.2$ & $-498.0 \pm 0.1$ & $-513.5 \pm 0.1$ & $-514.2 \pm 0.01$ & $-514.0 \pm 0.1$ & $-514.0 \pm 0.1$ \\
\hline
\end{tabular}

Table S2. Linewidth data for ${ }^{51} \mathrm{~V}$ NMR chemical shift signals of $\mathrm{V}_{\mathrm{A}}, \mathrm{V}_{\mathrm{B}}$ and $\mathrm{V}_{\mathrm{C}}$ in a $100 \mathrm{mM} \mathrm{NaVO}$ aqueous stock solution (of pH 3-8) and their corresponding reverse micellar (0.5 M AOT / isooctane) solution of $w_{0}=12,16$ and 20.

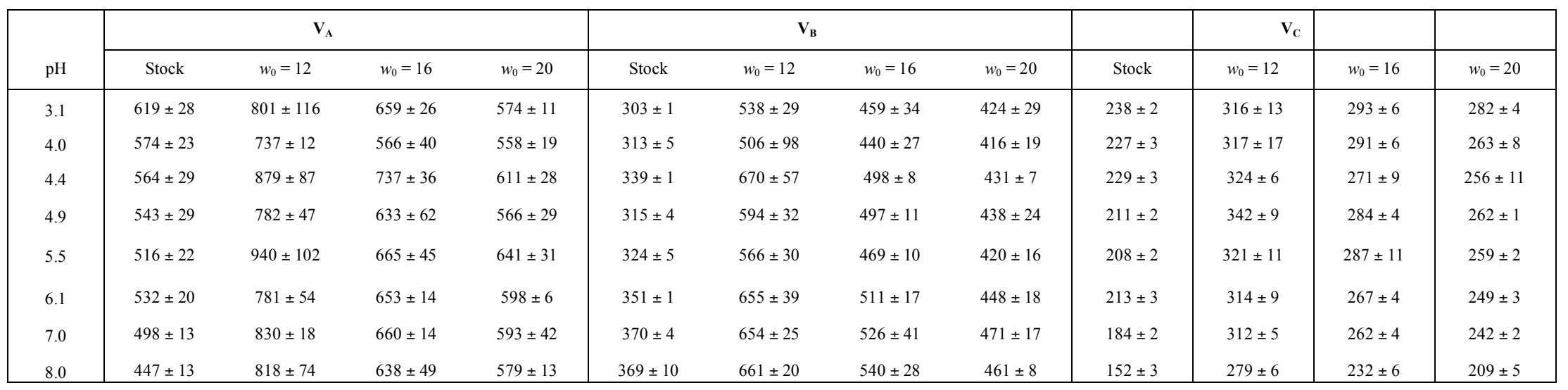

